\title{
International Year of Chemistry 2011
}

SCS

Division of

Industrial Chemistry
Popular Scientific Papers

Presented by the Division of Industrial Chemistry

\section{La chimie et l'industrie papetière}

Olivier Naef*

${ }^{\star}$ Correspondance: Prof. O. Naef, Ecole d'ingénieurs et d'architectes de Fribourg Institut de Chimie, Bd de Pérolles 80, CH-1705 Fribourg, Tél.: +41 264296705 E-mail: olivier.naef@hefr.ch

Abstract: This short paper presents the relationship between the pulp and paper industry and chemistry. The history of this industry is presented including current paper production and consumption statistics. The production of paper pulp is realized by chemically or mechanically separating cellulose fibres from wood and removing the unwanted lignin. This article concludes with an ecological aspect.

\section{Keywords: Chemistry $\cdot$ Industry $\cdot$ Paper $\cdot$ Production $\cdot$ Pulp}

\section{Introduction}

Ce siècle est marqué par une accélération du transfert de l'information. Si les moyens informatiques permettent d'amener rapidement un flot important de données, la lecture de ces données, malgré des annonces périodiques de «mort» du papier, passe encore très souvent par ce support nous arrivant tout droit de l'Antiquité (figure 1). Aujourd'hui encore, le papier et les cartons restent omniprésents dans notre monde. Si les pays industrialisés tentent de réduire leur consommation, les pays émergents augmentent dans des proportions plus importantes l'utilisation du papier.

Il est vrai que les documents sur papier comportent de nombreux avantages en tant que vecteur de l'information. Ils jouent en effet un double rôle de mémoire et d'instrument de lecture ne nécessitant aucune énergie. Les deux désavantages principaux du papier restent sa densité d'information par unité de volume ou de masse et les quantités de matières premières nécessaires à sa fabrication. Ainsi, un calcul très approximatif permet de déterminer la densité de l'information. Sur la base d'un quotidien standard, un poids de 122 g pour 40 pages représente une bonne

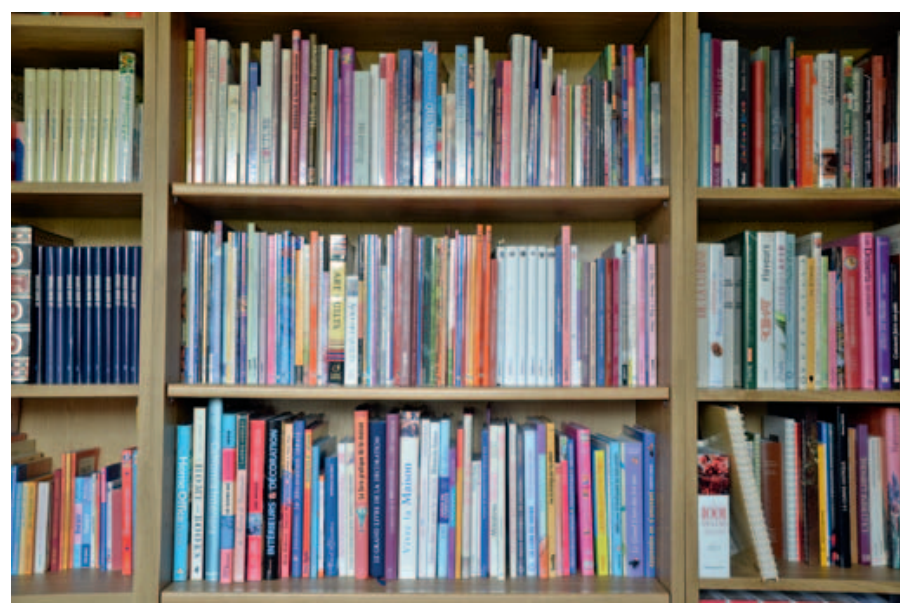

Figure 1. Omniprésence du livre et du papier dans notre monde. estimation. Avec une surface d'écriture de 1'300 $\mathrm{cm}^{2}$ par page et approximativement 420 caractères pour $24 \mathrm{~cm}^{2}$, un journal (sans image) contient 910'000 caractères soit presque 1 Mo (0.001 Go) pour $122 \mathrm{~g}$ ou un volume de $300 \mathrm{~cm}^{3}$. En comparant à un disque Blu-ray, qui dispose d'une mémoire de 27 Go pour une masse de $15 \mathrm{~g}$ et un volume de $11 \mathrm{~cm}^{3}$, l'augmentation de la densité est de plus de 700'000 par rapport au volume et 400'000 par rapport à la masse. Il est également important de noter qu'aujourd'hui, même si le journal papier est encore très présent, la concurrence face aux développements des supports de lecture électronique devient jour après jour plus féroce. Il serait très intéressant de suivre ces deux marchés au cours des 10-20 prochaines années avec l'avancée technologique des écrans flexibles tels que l'écran de LG. ${ }^{[1]}$ Néanmoins, les journaux ne représentent que $13 \%$ de la consommation mondiale de la pâte à papier. Les cartons forment à eux seuls $56 \%$ de cette consommation et les papiers d'impression concernent $31 \%$.

\section{Le papier de l'Antiquité à nos jours}

L'histoire du papier débute en Chine. ${ }^{[2]}$ C'est Tsaï Loun, ministre chinois de l'agriculture, qui, aux environs du $\mathrm{III}^{\mathrm{e}}$ siècle avant Jésus-Christ, décrit la fabrication du papier à partir de fibres de bambous, d'écorces de mûriers, de lins et de chanvres. Cet art devra attendre le VIII ${ }^{\mathrm{e}}$ siècle pour arriver dans le monde arabe qui, très rapidement, l'utilise pour propager l'Islam. Au fur et à mesure des guerres et de l'avancée des Arabes en Occident, cette technique se développe et arrive en Europe entre le XII ${ }^{\mathrm{e}}$ siècle et le XIV siècle. Le XV siècle marque un tournant de l'histoire du papier en Europe avec l'invention de l'imprimerie par Johannes Gutenberg; les Protestants utilisent alors cette nouvelle technique pour répandre la Bible.

L'industrialisation de la fabrication du papier débute au XIX ${ }^{\mathrm{e}}$ siècle. La première étape est franchie par Nicolas Robert lors de la Révolution française avec la machine à fabriquer le papier «à grande étendue». La deuxième étape correspond au remplacement des chiffons, dont l'approvisionnement devient critique, par de la pâte à papier composée d'un mélange de fibres de bois et d'eau. L'invention revient à Friedrich Gottlob Keller qui fabrique cette pâte mécaniquement à l'aide d'une meule (brevet 1844). Aristide Bergès, après avoir apporté différentes améliorations, devient un des grands promoteurs de cette technique. Parallèlement, au cours de cette deuxième moitié du XIX ${ }^{\mathrm{e}}$ siècle, les chimistes mettent au point le procédé par voie chimique, en opposition au procédé mécanique. En 1834, le chimiste Anselme Payen, qui étudie la composition chimique du bois, isole la cellulose sous la forme d'une substance fibreuse blanche dont les propriétés sont similaires à celles du coton. Les procédés chimiques développés permettent d'extraire la cellulose sous la forme d'une pâte à papier.

Comme présenté à la figure 2 , la consommation de papier a triplé au cours de ces quatre dernières décennies pour atteindre un peu moins de 400 millions de tonnes en 2010. Si la quantité pour les journaux a doublé, celle des papiers d'impression a plus que quadruplé pendant que celle des cartons triplait depuis les années 1970. L'augmentation de cette consommation est également très divergente en fonction des pays. ${ }^{[4]}$ Les statistiques 2009 


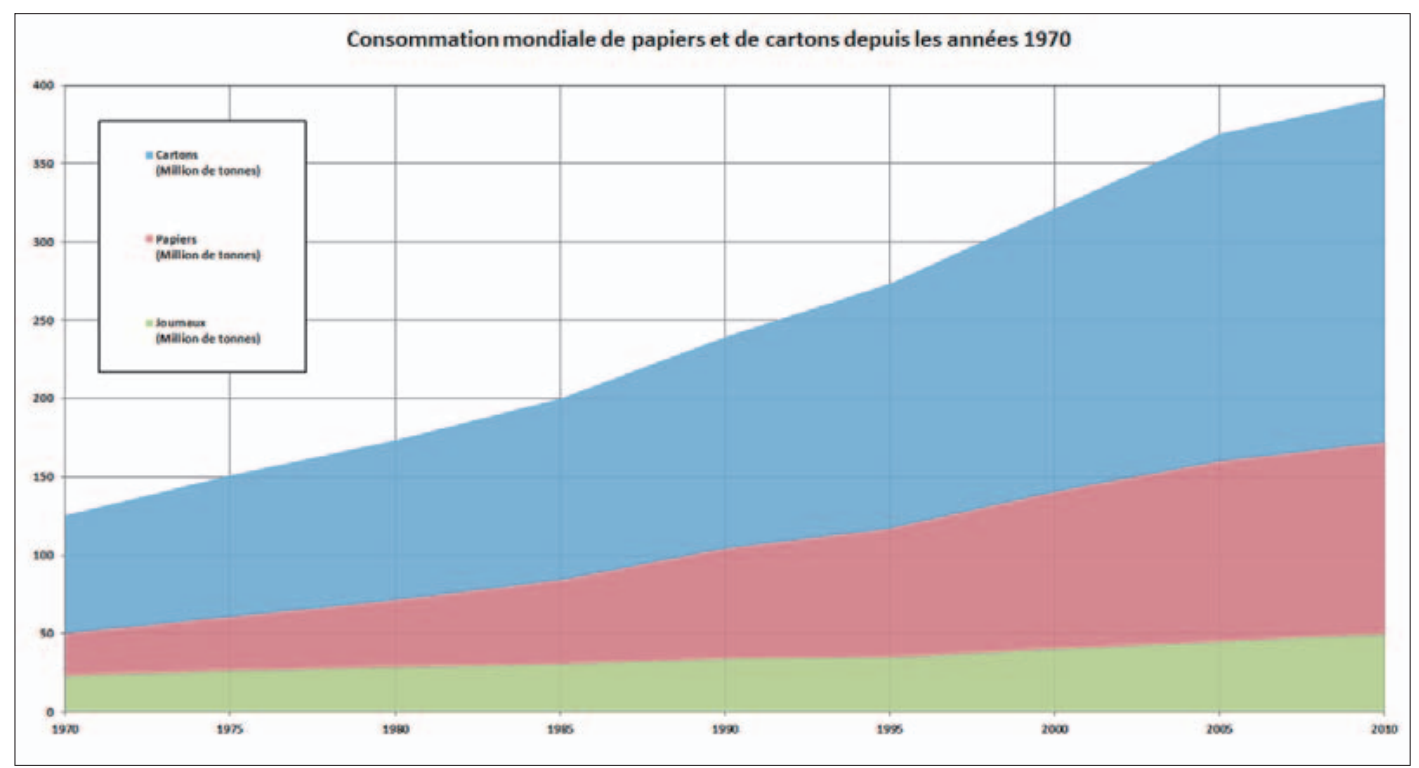

Figure 2. Consommation mondiale de papiers et de cartons depuis les années 1970 à nos jours (données en millions de tonnes ${ }^{[3]}$. de la «European Pulp and Paper Industry»[5] montrent que ses membres (Europe) ont fortement réduit leur production et leur consommation. Pour l'année 2009, la production de pâte à papier à partir de bois a été d'un peu moins de 36 millions de tonnes, soit une réduction de $13.5 \%$ par rapport à l'année précédente. La production a été réalisée pour un peu moins de $68 \%$ par voie chimique et $30.5 \%$ par voie mécanique et semi-chimique. Parallèlement, 45 millions de tonnes ont été produites à base de papier recyclé (réduction de $8 \%$ par rapport à 2008), pour un total de 88.7 millions de tonnes.

\section{Fabrication de la pâte à papier}

Une feuille de papier est principalement constituée de fibres végétales issues du bois. ${ }^{[6]} \mathrm{D}$ 'autres végétaux comme le lin, le coton, le chanvre, ... permettent d'obtenir les propriétés particulières du papier. Pour des applications spécifiques, comme les billets de banque ou les filtres, des fibres artificielles ou animales peuvent également être utilisées. Trois classes de propriétés permettent de classifier le papier: les propriétés de résistance mécanique, les propriétés optiques et les propriétés de texture. Les fibres de cellulose ont des tailles différentes en fonction de leurs provenances. Les adjuvants chimiques ajoutés en cours du procédé permettent de modifier les propriétés du papier. Les éléments minéraux comme le kaolin, le carbonate de calcium, le dioxyde de titane, ... permettent de modifier les propriétés optiques du papier.

Le bois est constitué essentiellement de polysaccharides comme la cellulose (figure 3, environ $45 \%$ ) et l'hémicellulose, ainsi que de la lignine ${ }^{7]}$ (polymère formé dans des proportions diverses à partir de monomères de l'alcool coumarylique, de l'alcool coniférylique et de l'alcool sinapylique, environ 25\%). La fabrication de la pâte à papier consiste à extraire les fibres du bois. La voie mécanique pure ne supprime pas la lignine (rendement de $95 \%$ ). Le papier a une faible résistance au vieillissement et les propriétés mécaniques ne sont pas optimales. L'application d'un traitement thermique à la vapeur permet d'améliorer les propriétés mécaniques finales. Cette voie thermique peut être complétée par un traitement chimique ${ }^{[7]}$ à l'aide de sulfite de sodium et de soude qui favorise le défibrage et rend la lignine plus hydrophile. Depuis les années 1990, un nouveau procédé utilisant le peroxyde d'hydrogène en milieu basique s'est développé.

Le procédé alcalin, ${ }^{[7]}$ appelé également procédé au sulfate ou encore procédé kraft, consiste à dissoudre complètement la

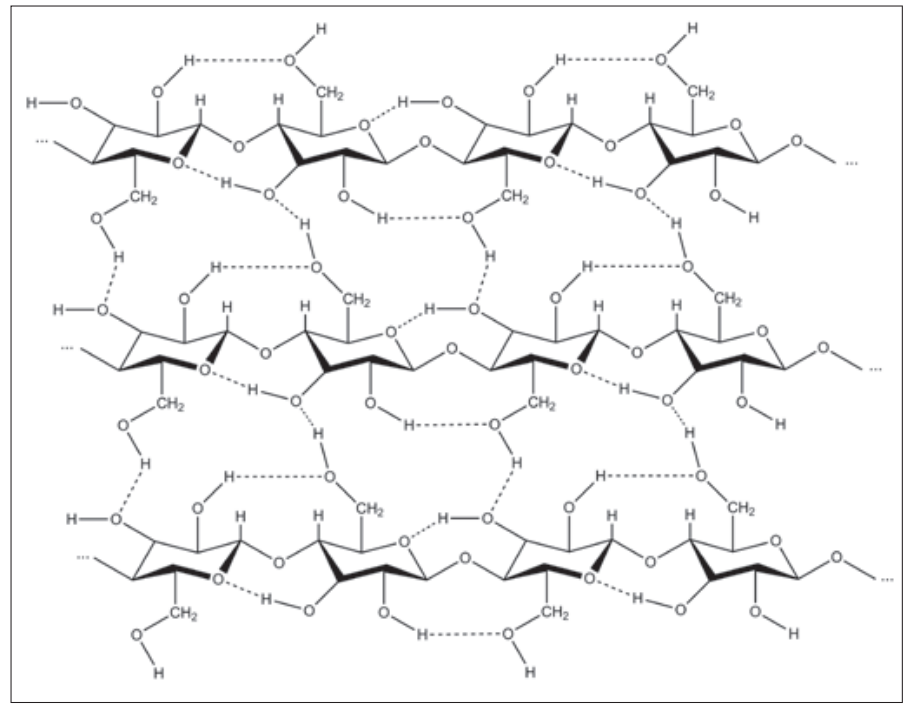

Figure 3. Cellulose correspondant à une chaîne linéaire D-Glucose.

lignine à l'aide d'une cuisson à $170{ }^{\circ} \mathrm{C}$ en présence de soude et de sulfure de sodium. Le sulfure de sodium est obtenu par combustion à partir du sulfate de sodium $\left(\mathrm{Na}_{2} \mathrm{SO}_{4}\right)$. Au cours de la cuisson, le $\mathrm{Na}_{2} \mathrm{SO}_{4}$ est hydrolysé en soude, $\mathrm{NaHS}$ et $\mathrm{H}_{2} \mathrm{~S}$. Les composés soufrés réagissent avec la lignine pour former des thiolignines solubles. Il est possible de réduire le temps de cuisson en ajoutant comme catalyseur des composés quinoniques comme l'anthraquinone. Ce procédé implique une régénération de la plus grande partie des produits chimiques. Les déchets organiques sont quant à eux brûlés pour former de la vapeur utilisée pour la production électrique. Le seuil de rentabilité s'établit aux environs de 500 tonnes de pâte à papier produites par jour.

Dans le cas des bois résineux, un procédé acide ${ }^{[7]}$ ou au bisulfite est également possible. La lignine réagit avec de l'anhydride sulfureux libre provenant de l'hydrogénosulfite de sodium, d'ammonium ou de magnésium (préféré à cause de sa facilité de régénération).

En dehors de certains papiers ou cartons, la pâte à papier écrue doit être blanchie. La couleur correspond à de la lignine résiduelle. Le blanchiment a pour but soit de retirer complètement la lignine sans endommager les polysaccharides, soit de modifier les groupements colorés ou chromophores présents. Le chlore est utilisé jusque dans les années 80 en tant qu'agent de 
blanchiment. Celui réagit avec la lignine sans affecter la cellulose. Chaque étape de blanchiment est suivie d'un lavage. Malgré son prix, le $\mathrm{ClO}_{2}$ remplace petit à petit le chlore pour des raisons environnementales. Parallèlement, le Prof. André Robert découvre le moyen de blanchir les fibres cellulosiques avec de l'oxygène, tout en protégeant la cellulose avec des sels de magnésium. Cette technique est utilisée aujourd'hui encore dans plus de la moitié des sites de production. Au cours des années 1990, le développement de l'utilisation de l'ozone ${ }^{[8]}$ permet une réduction de la charge polluante des effluents du blanchiment de la pâte à papier.

Avant la fabrication du papier ou du carton sur la machine à papier, la préparation de la pâte ${ }^{[9]}$ constitue l'ultime étape permettant de fournir une matière première conforme au cahier des charges. Les fibres doivent être bien dispersées, propres, décontaminées. Des additifs appropriés comme les charges dans la masse, les produits de collage, les agents de rétention ou des colorants et agents de fluorescence sont ajoutés.

Les charges dans la masse ou adjuvants chimiques, comme discuté ci-dessus, peuvent représenter jusqu'à $30 \%$ de la masse finale du papier. Ces charges servent à améliorer l'impression et les propriétés optiques du papier (opacité, blancheur et lissé). Les caractéristiques mécaniques du papier diminuent avec le taux de charges. Les produits de collage servent à transformer le caractère hydrophile du papier (papier buvard par exemple) en un matériau hydrophobe. La résine de synthèse alkyl ketène dimère, par exemple, est utilisée en milieu neutre. Elle est ajoutée par réaction chimique sur la cellulose grâce aux groupements hydroxyles de celle-ci. Les agents de rétention servent de système de retenu pour les particules solides de la pâte à papier au cours de la phase d'égouttage. L'action de ces agents peut être apparentée à une coagulation et/ou une floculation. Des produits, comme l'amidon par exemple, sont encore ajoutés pour améliorer les propriétés mécaniques du papier. Les autres additifs permettent d'améliorer ou d'éviter des problèmes lors du passage de la pâte à papier dans la machine à papier.

\section{Enjeux écologiques et économiques}

L'industrie papetière est encore souvent citée comme très polluante de notre environnement et responsable de la déforestation. Cette idée de déforestation est même pointée du doigt par la Fédération des consommateurs (http://www. $f s c$-schweiz.ch/fr/ les-produits-fsc/fsc-papier, visité le 25.04.11): l'article parle de pillage des forêts pour la production des 360 millions de tonnes de papier produites. Le Canada utilise pour sa production plus de $50 \%$ de copeaux de bois, de bois de rebut et d'autres résidus $\mathrm{du}$ bois des scieries (http://www.thecanadianencyclopedia.com, visité le 25.04.11). La France n'utilise pratiquement que du bois provenant de coupes éclaircies et de déchets de scierie. Il est d'ailleurs important de rappeler que la France produit la moitié de sa pâte à papier avec du papier recyclé, réduisant d'autant les quantités d'eau et d'électricité nécessaires. De plus, lorsque le papier n'est pas récupéré, il peut être brûlé, offrant un substitut à l'énergie fossile. Le rapport 2009 de Copacel ${ }^{[10]}$ montre clairement les efforts réalisés par l'industrie papetière française dans le domaine de l'écologie. Le gouvernement du Canada a annoncé le 14 mars de cette année des investissements de l'ordre de 88 millions de dollars pour favoriser la production d'énergie renouvelable dans les usines de pâte à papier.

L'industrie papetière représente $2.5 \%$ de l'industrie mondiale. Il est également intéressant de noter que cette industrie n'est pratiquement pas associée par le grand public à l' «industrie chimique», même si les procédés font de plus en plus intervenir des opérations chimiques.

\section{Conclusion}

L'industrie papetière fournit avec l'aide de la chimie des papiers et des cartons. Comme toute grande industrie, les changements sont plus lents, car ils nécessitent souvent d'importants investissements. Pourtant cette industrie a su constamment innover afin de relever les différents défis technologiques. Aujourd'hui encore, la recherche se poursuit tant dans le domaine du blanchiment que du recyclage des eaux utilisées. De nombreuses industries papetières travaillent non seulement dans une vision économique mais également dans une vision de développement durable.

Received: May 5, 2011

[1] M. Williams, 'Samsung, LG Show Flexible OLED, E-paper Screens', IDG News (online), 2010 (accessed 23.04.2011).

[2] G. Coste, 'Le papier, la belle histoire', Revue de l'ingénieur INPG $\mathbf{2 0 0 3}$, 03-4, 28-29.

[3] D. Lachenal, 'Industrie papetière mondiale: croissance prévue jusqu'en 2015', Revue de l'ingénieur INPG 2003, 03-4, 37.

[4] B. Galembert, 'Pulp and paper markets and forecasts', Confederation of European Paper Industries: Brussel, 2009, pp 1-38.

[5] CEPI Key Statistics 2009: European Pulp and Paper Industry, Confederation of European Paper Industries: Bruxelles, 2009; pp 1-28.

[6] C. Voillot, 'Voyage au cœur du papier', Revue de l'ingénieur INPG $\mathbf{2 0 0 3}$, 03-4, 30-31.

[7] M. Petit-Conil, 'Procédés papetiers - Fabrication des pâtes', Techniques de l'ingénieur 1999.

[8] J.-C. Hostachy, 'Réduction de la charge polluante des effluents du blanchiment de la pâte à papier par un traitement à l'ozone', Institut national polytechnique de Grenoble, Grenoble, 1994.

[9] A. Lemaître, 'Procédés papetiers - Préparation des pâtes', Techniques de l'ingénieur 2003.

[10] COPACEL L'Industrie Papetière - Rapport Développement Durable, Confédération Française de l'Industrie des Papiers, Cartons et Celluloses, Paris, 2009 